DOE / PC92544-12

VAPOR PRESSURES AND HEATS OF VAPORIZATION OF PRIMARY COAL TARS

E. M. SUUBERG (PRINCIPAL INVESTIGATOR)

V. OJA (GRADUATE STUDENT)

M. HONEYCUTT, M. NEVITT and J. LIANG

(UNDERGRADUATE SUMMER INTERNS)

W.D. LILLY (STAFF)

DIVISION OF ENGINEERING

BROWN UNIVERSITY

PROVIDENCE, RI 02912

TEL. (401) 863-1420

QUARTERLY TECHNICAL PROGRESS REPORT

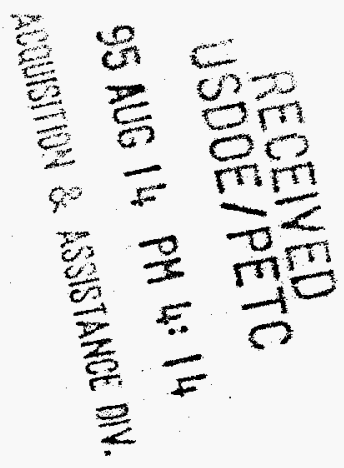

1 APRIL, 1995- 30 JUNE, 1995

PREPARED FOR:

U. S. DEPT. OF ENERGY

PITTSBURGH ENERGY TECHNOLOGY CENTER

P.O. BOX 10940

PITTSBURGH, PA 15236

DR. KAMALENDU DAS, METC, MORGANTOWN , WV TECHNICAL PROJECT OFFICER

"US/DOE Patent Clearance is not required prior to the publication of this document" 


\section{DISCLAIMER}

Portions of this document may be illegible in electronic image products. Images are produced from the best available original document. 


\section{General Background}

As the world continues to deplete its petroleum reserves, then heavy crude oil, coal liquids, and other heavy fossil fuels may be required to meet the world energy needs. Heavy fossil fuels contain molecules that are large and more aromatic and that contain more heteroatoms than those found in liquid crude oil.

There is also significant current interest in general area of coal pyrolysis, particularly with with respect to comprehensive models of this complicated phenomenon. This interest derives from central role of pyrolysis in all thermally driven coal conversion processes - gasification, combustion, liquefaction, mild gasification, or thermal benefication. There remain several key data needs in these application areas. Among them is a need for more reliable correlation for prediction of vapor pressure of heavy, primary coal tars. Such information is important in design of all coal conversion processes, in which the volatility of tarry products is of major concern.

The vapor pressure correlations that exist at present for coal tars are very crude and they are not considered reliable to even an order of magnitude when applied to tars. Sophisticated general correlative approaches are slowly being developed, based upon group contribution methods, or based upon some key functional features of the molecules. These are as yet difficult to apply to coal tars. The detailed group contribution methods, in which fairly precise structural information is needed, do not lend themselves well for application to very complex, poorly characterized coal tars. The methods based upon more global types of characterizations have not yet dealt much with the question of oxygenated functional groups. In short, only very limited correlations exist, and these are not considered reliable to even an order of magnitude when applied to tars.

The present project seeks to address this important gap in the near term by direct measurement of vapor pressures of coal tar fractions, by application of well-established techniques and modifications thereof. The principal objectives of the program are to: 1) obtain data on the vapor pressures and heats of vaporization of tars from a range of ranks of coal, 2) develop correlations based on a minimum set of conveniently measurable characteristics of the tars, 3) develop equipment that would allow performing such measurements in a reliable, straightforward fashion. 


\section{Result from this Quarter}

During this quarter, the project benefitted from the participation of two DOE summer interns, Misti Honeycutt and Mark Nevitt of Cumberland College and one Brown undergraduate, Mr. Jim Liang. These students worked alongside Mr. Vahur Oja in the laboratory. In the initial phases of their participation, some time needed to be devoted to their training in the measurement techniques. Thereafter, they became a valuable asset to the project.

At this time, both the gas saturation method and the Knudsen effusion methods are fully reliable methods. These have both been described in detail in earlier quarterlies, and these details will not be repeated here. Figure 1 shows a comparison of the results obtained using the two methods, and a comparison with the mean of the data from the literature. It can be seen that there is generally good agreement between the two techniques, and that both give results that agree well with published values. It should be recalled that for measurements on coal tars, as is ultimately the objective in this project, this level of accuracy is certainly acceptable because of the uncertainties that will be introduced as a result of working with complicated mixtures. We feel that the effusion method is at present slightly more accurate, for reasons not yet fully understood. This judgement is based upon several factors, including data scatter and agreement with published literature.

Figure 2 shows the most recent results obtained with $99+\%$ purity anthracene, using the effusion method. Also shown are the results calculations of the vapor pressure at the same temperatures as were used for measurement, based upon reported correlations. It can be seen that our results confirm the majority of results already present in the literature.

Figures 3 and 4 show the results obtained for naphthacene and pentacene, using the effusion method. The former is of $98 \%$ purity, and the latter is of reagent grade, of unestablished purity. Again, outstanding agreement has been obtained with the few data in the literature on these polycyclics. It should be noted that these measurements extended the range of measurement temperature up to $212^{\circ} \mathrm{C}$, showing that the measurement technique is reliable to at least these temperatures.

Coal tars are, of course, not pure single-component materials. Rather, they are 
very complicated mixtures containing many hundreds or thousands of components. In recognition of the fact that it would be necessary to deal with mixtures, some work has been performed on mixtures of pure components. An example is shown in Figure 5, for a mixture of anthracene and perylene. The anthracene was of $99+\%$ purity and the perylene was of reagent grade, but not tested for purity.

It may be seen from Figure 5 that the mixture of these two polycyclic aromatics is nearly ideal, and exhibits a vapor pressure quite near to that predicted by Raoult's law:

$$
P_{\text {tot }}=x_{a} P_{a}{ }^{0}+x_{b} P_{b}{ }^{o}
$$

where $x_{i}$ refers to the mol fraction of $i$ in the mixture, and $P_{i}{ }^{\circ}$ is the pure $i$ vapor

pressure. Clearly the vapor pressure of the mixture is dominated by the vapor pressure of pure anthracene, since the perylene is practically non-volatile in comparison.

The near-ideality exhibited in Figure 5 cannot generally be expected for all components of coal tar. Coal tars are not pure polycyclic aromatic hydrocarbons, but may contain significant numbers of heteroatoms. An example of what can happen in heteroatomic systems is seen in Figure 6, for a mixture of 1-hydroxypyrene and phenanthridine. In this case, the mixture is seen to be highly non-ideal, and the deviation from the Raoult's law prediction is substantial (about an order of magnitude). The fact that the mixture shows a much lower than predicted vapor pressure is not surprising, insofar as the components were deliberately selected to show a strong electron donoracceptor type of interaction. The methods to be used for describing the coal tar mixtures will be described in the next quarterly report.

During the latter part of this quarter, attention was turned to the preparation of wellcharacterized coal tars. The technique involved separation of coal tars on a preparativescale gel permeation column, so as to yield narrow molecular weight fractions. Unfortunately, the column had become too contaminated through years of use, and the separations were too poor to be of use in this study. Thus this part of the project had to be delayed, pending replacement of the column.

\section{Plans for Next Quarter}

Work continues on the tar preparation and characterization equipment. Steps are being taken to obtain a new preparative scale column. The project budget will not permit 
purchase of a new commercial column, since these typically cost several thousands of dollars. Instead, a source of bulk packing material is being sought.

Meanwhile, work on pure compounds, mixtures, and other tars not requiring GPC separation continues, until the new column is available.

\title{
5.0 References
}

DeKruif, C.G.,J. Chem. Thermodynamics, 1980, 12, 243.

"Handbook"- Stephenson,R.M and Malanowski, S.", Handbook of the Thermodynamics of Organic Compounds," Elsevier, Amsterdam, 1987.

Hansen, P.C. and Eckert, C.A., J. Chem. Eng. Data, 1986, $31,1$.

Kelley, J.D. and Rice, F.O., J. Phys. Chem., 1964, 68, 3795.

Macknic, A.B. and Prausnitz, J.M., J. Chem. Eng. Data, 1979, 24, 175.

Sonnefeld, W.J. and Zoller, H., Anal. Chem., 1983, 55, 275.

\section{DISCLAIMER}

\begin{abstract}
This report was prepared as an account of work sponsored by an agency of the United States Government. Neither the United States Government nor any agency thereof, nor any of their employees, makes any warranty, express or implied, or assumes any legal liability or responsibility for the accuracy, completeness, or usefulness of any information, apparatus, product, or process disclosed, or represents that its use would not infringe privately owned rights. Reference herein to any specific commercial product; process, or service by trade name, trademark, manufacturer, or otherwise does not necessarily constitute or imply its endorsement, recommendation, or favoring by the United States Government or any agency thereof. The views and opinions of authors expressed herein do not necessarily state or reflect those of the United States Government or any agency thereof.
\end{abstract}




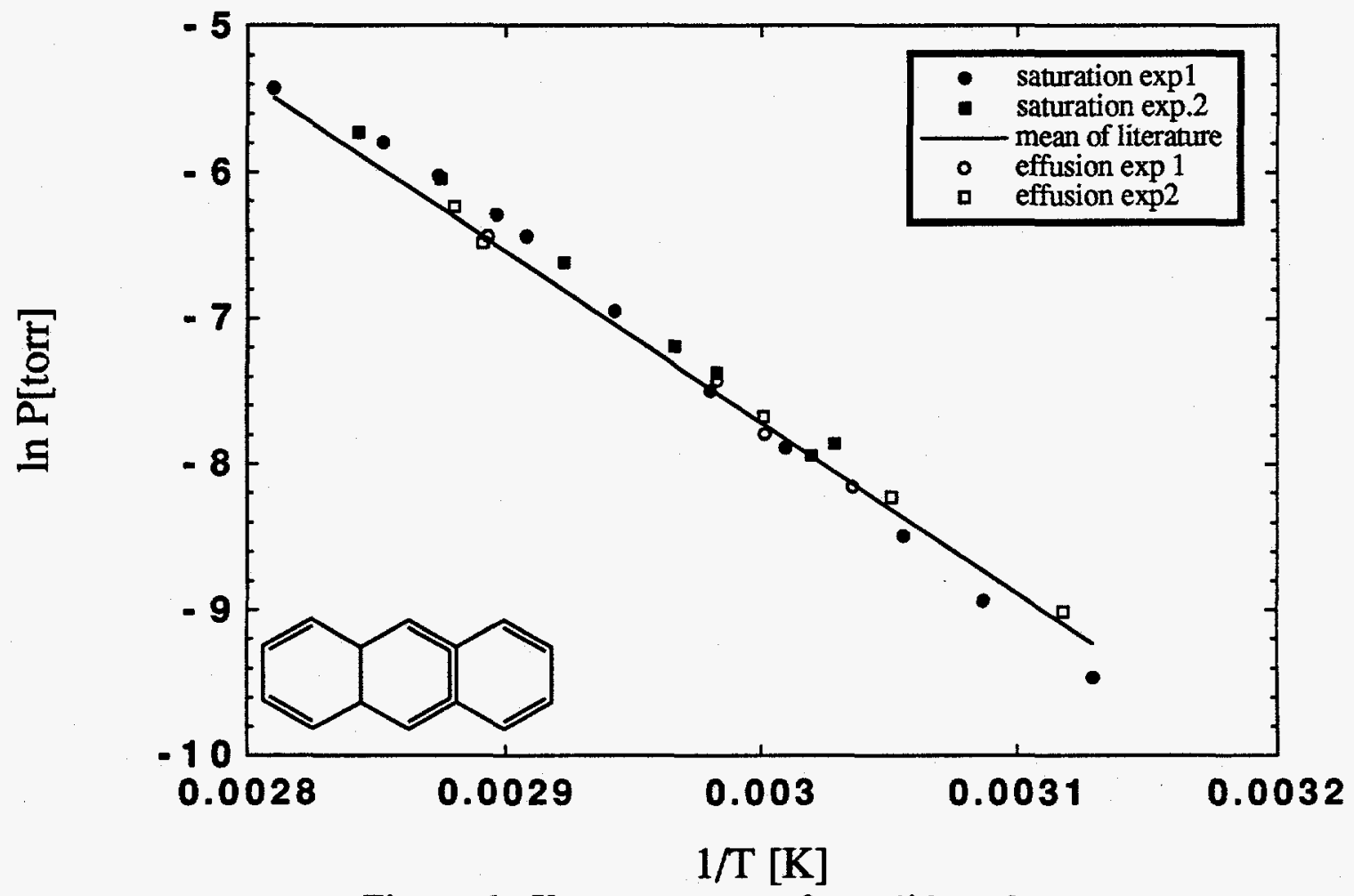

Figure 1. Vapor pressure for solid anthracene.

A comparison of the results obtained using the effusion and saturation techniques. 


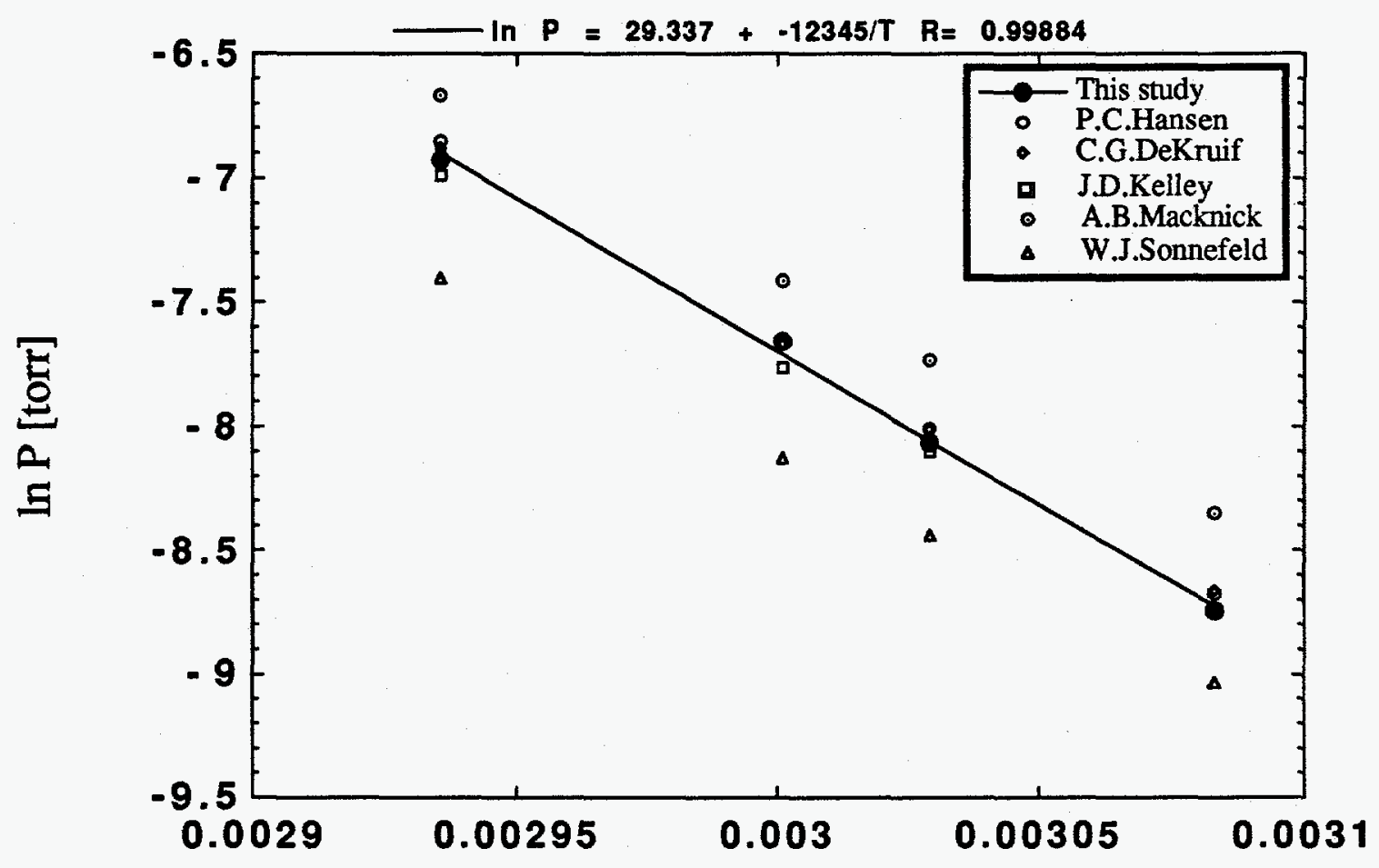

Figure 2. Effusion method applied to solid anthracene. Solid points are from this study, open are from references. 


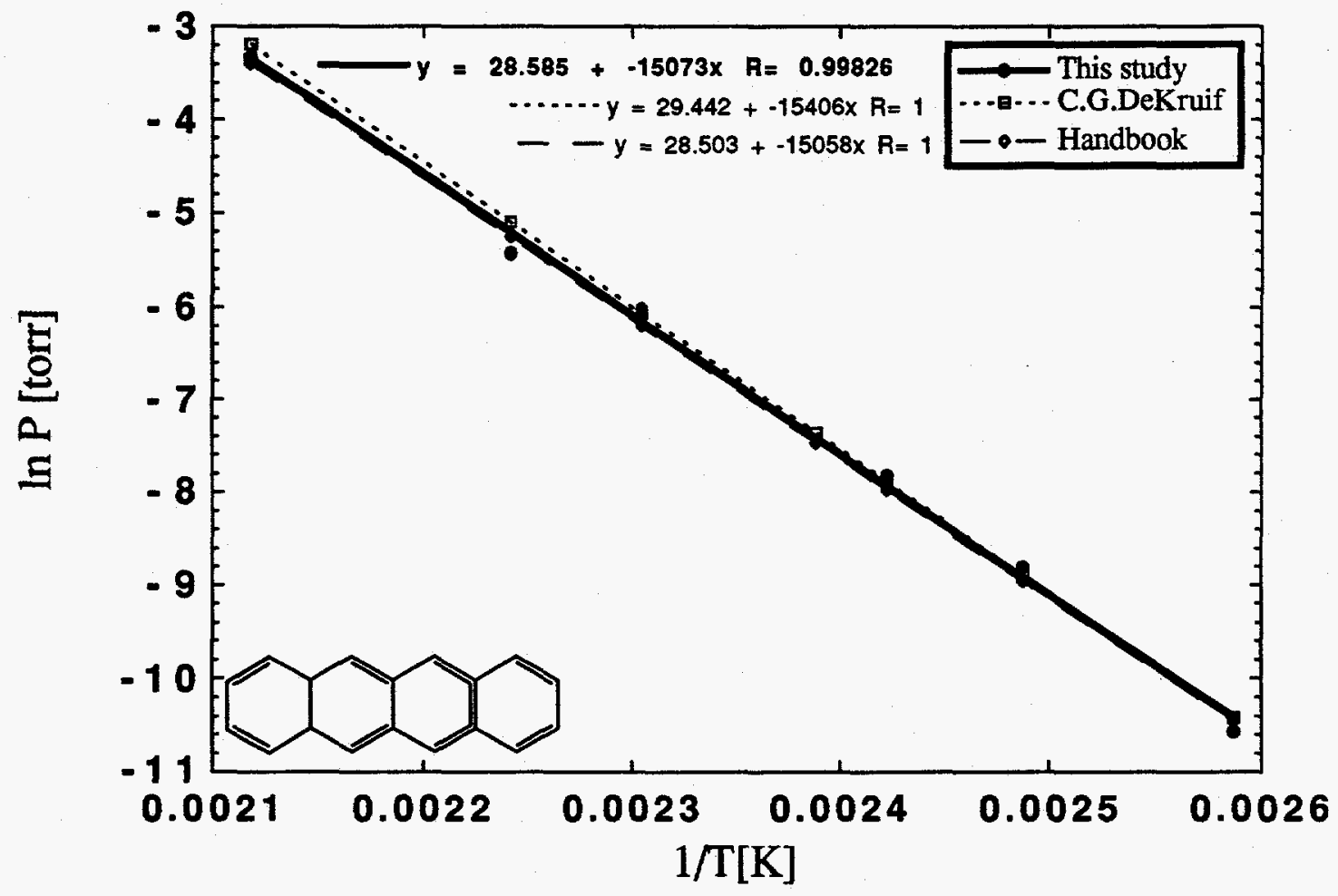

Figure 3. Effusion method applied to solid naphtacene. The solid points are from this study, and the open are from the literature. 


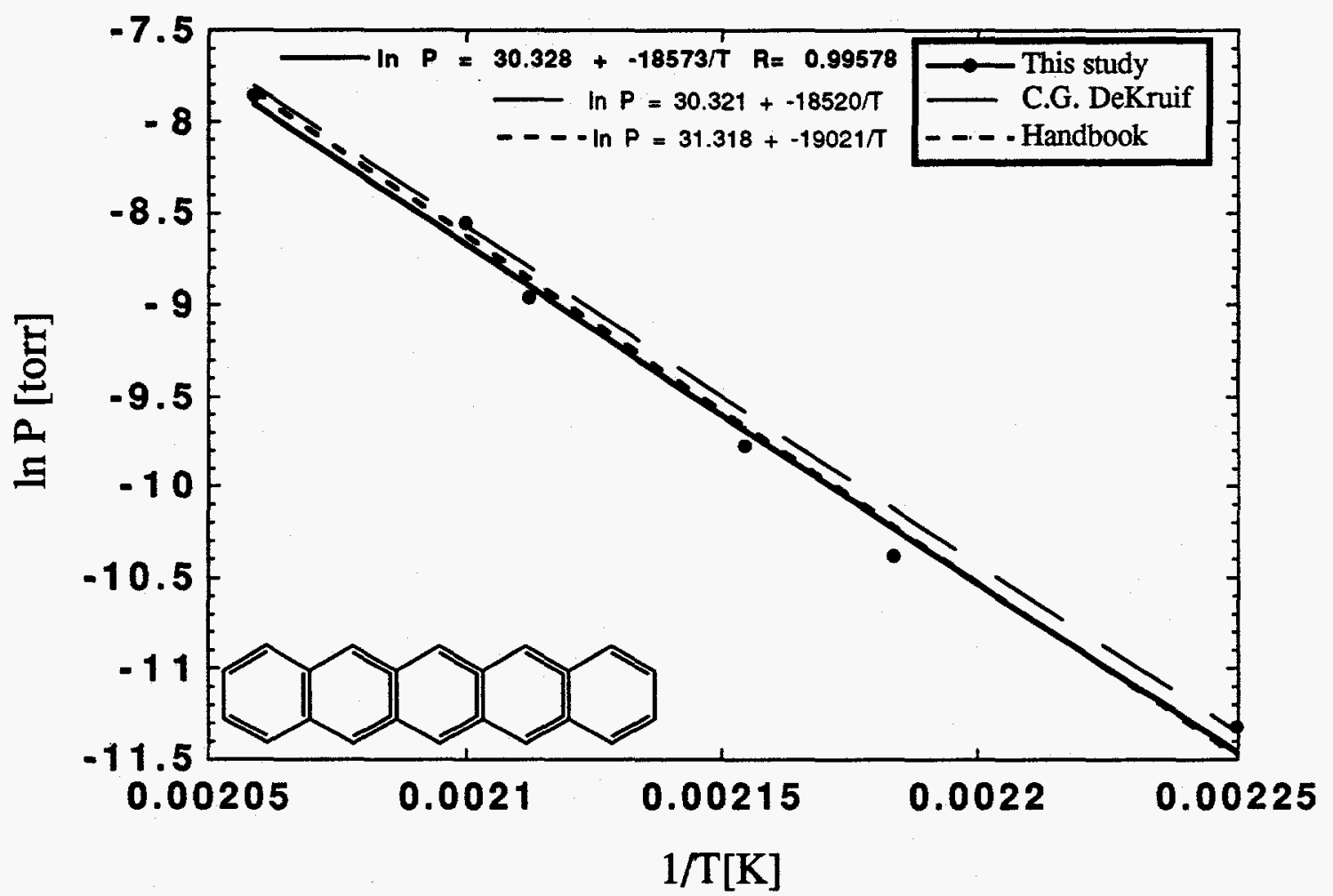

Figure 4. Effusion method applied to solid pentacene. 


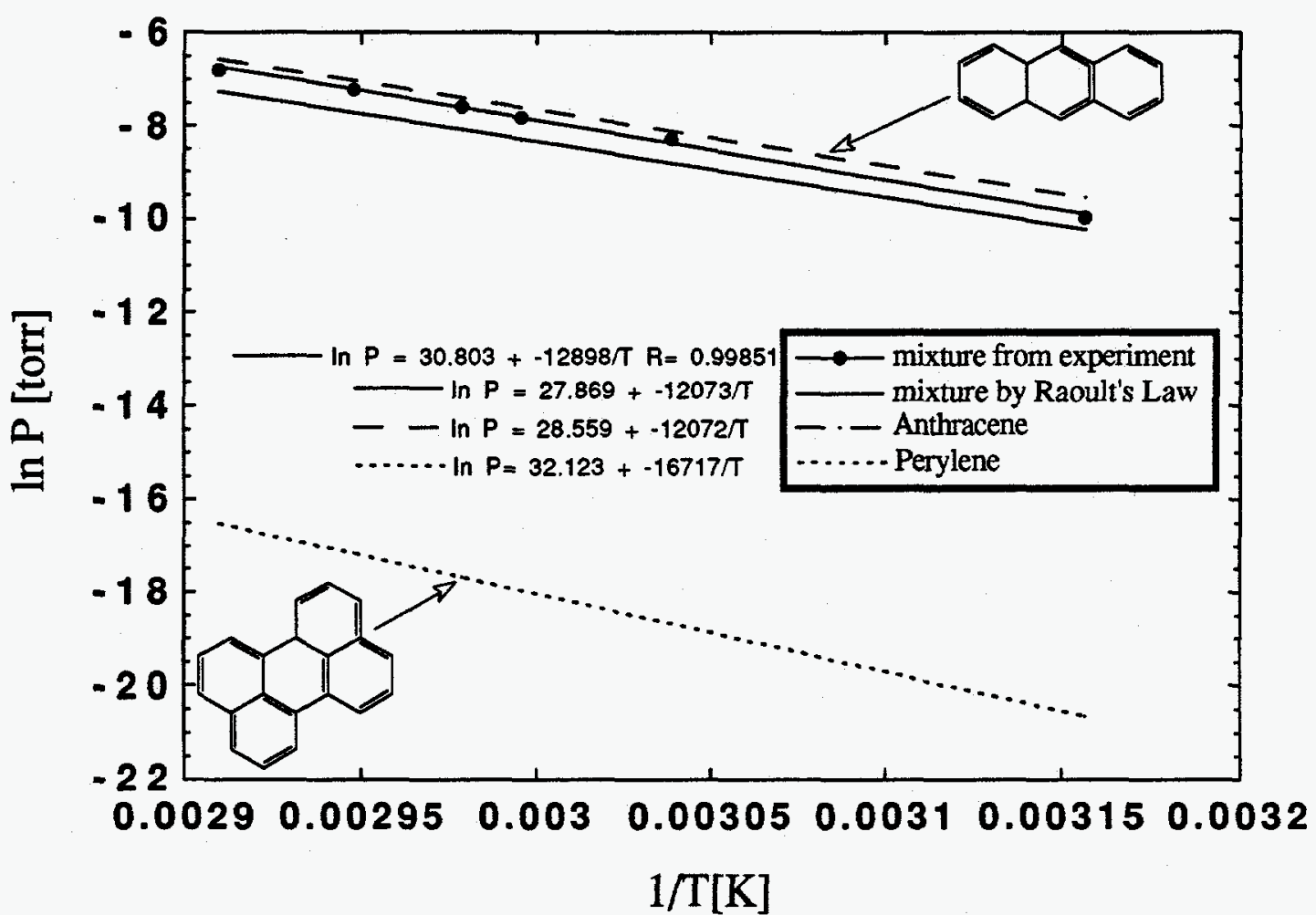

Figure 5. Effusion method applied to a mixture of anthracene $(50 \%)$ and perylene (balance). Data for pure components shown for comparison. 


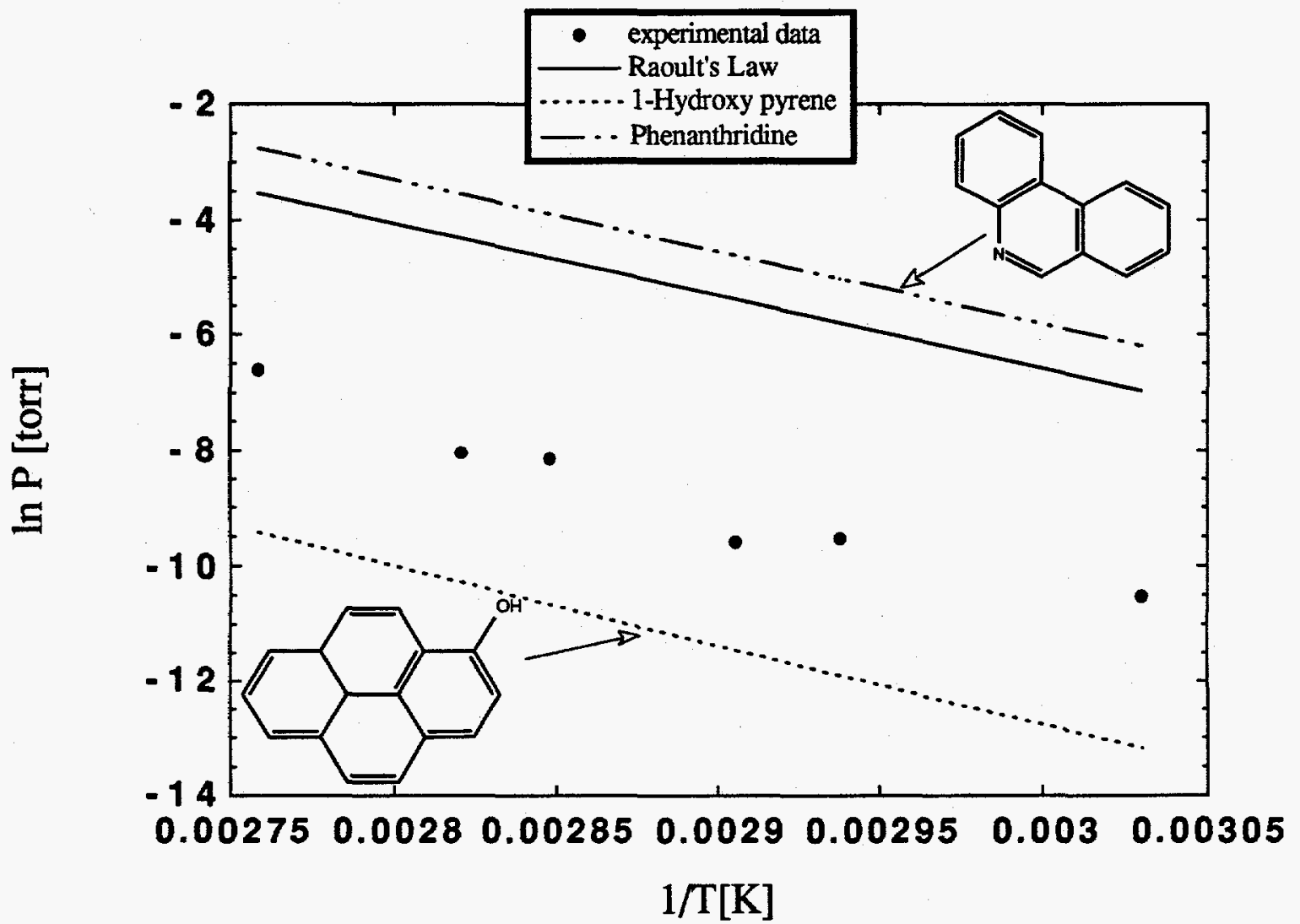

Figure 6. Effusion method applied to a mixture of 1-hydroxypyrene $(54 \%)$ and phenanthridine (balance). Data for pure components shown for comparision. 